\title{
Abducens Nerve Disorder
}

National Cancer Institute

\section{Source}

National Cancer Institute. Abducens Nerve Disorder. NCI Thesaurus. Code C27593.

A non-neoplastic or neoplastic disorder affecting the abducens nerve (sixth cranial nerve). 